\title{
A study of the effect of short-term relaxation on the EIS test technique for EV battery cells
}

\begin{abstract}
Electrochemical impedance spectroscopy (EIS) is a powerful tool employed to investigate the fundamental electrochemical parameters of a $\mathrm{Li}$-ion battery cell. Its effectiveness has established it as a core method to study electrochemical behavior of batteries in laboratory environments. An industry-wide interest currently exists to employ EIS as part of battery management systems (BMSs) for state of charge, internal temperature and state of health measurements. The major issue of employing EIS for online measurement as part of BMS is the reliability. In this work, it is shown that not all the parameters extracted by the EIS technique are reliable when measurement is performed shortly after charge/discharge. While pure Ohmic resistance was found to be consistent, the change transfer resistance and double layer capacitance are not reproducible. Therefore, only $R_{o}$ can be reliably employed for the fast online measurement by BMS.
\end{abstract}

Keywords: electrochemical impedance spectroscopy, battery cells

\section{Introduction}

Intrinsic properties of lithium-ion (Li-ion) batteries, such as high energy, high power density, long cycle life and low self-discharge rate, have enabled their use in electric vehicle (EV) applications. Equivalent circuit models (ECMs) are commonly used by the battery management system (BMS) of an EV to estimate battery performance in real operating scenarios. In addition, investigation of the electrochemical processes, i. e., Li-ion diffusion in the electrolyte, migration through the solid electrolyte interface (SEI) layer, charge transfer through the electrode/electrolyte interface and solid state diffusion in the bulk of the active material can be achieved by using electrochemical impedance spectroscopy (EIS). As each process has a different time constant, time domain or frequency of excitation [1, 2], EIS allows for the separation of most of these processes [3]. Therefore, EIS always interests academic and industrial researchers as a diagnostic and research tool. A standard EIS test within a frequency range from $10 \mathrm{kHz}$ to $10 \mathrm{mHz}$ and 10 frequency points per decade of frequency, as commonly employed in the literature, requires around 10 minutes. However, if the number of frequency points is reduced, the test duration can be aggressively reduced to seconds. This potential reduction of test duration has allowed EIS to be incorporated as an integral part

Anup Barai, Andrew McGorden, Paul Jennings, WMG, University of Warwick, Coventry, CV4 7AL, United Kingdom, e-mail: A.Barai@warwick.ac.uk

https://doi.org/10.1515/9783110558920-004 
of BMS to estimate the state of charge (SoC) and state of health (SoH) in EVs [4-6]. For such onboard EIS measurement systems (incorporated as part of the BMS), a short measurement time is a key requirement. However, fast measurement implies that the cells will be measured very shortly after cycling. Recently the cell relaxation period has been identified as a source of impedance variation $[7,8]$. When the charge or discharge process of the cell is stopped, the polarization of the cell ceases and as a result, the cell impedance and voltage of the cell change, which is referred to as relaxation of the cell and which is mainly caused by relaxation of ions within the double layer, electrolyte ionic concentration gradient redistribution and solid state diffusion of lithium atoms within the bulk of the materials. This lead to change of cell impedance in a couple of hours after the charge/discharge event. In [8] it has been shown that a measurable change of impedance will continue until 4 hours after a discharge event. This research indicates potential hurdles to get repeatable and reproducible results from EIS tests when employed shortly after cycling. Currently there is no research present in the literature showing how reliable and reproducible the EIS tests are when performed immediately after charge/discharge. This research focuses on the impedance variation of the cell within a short period (0-10 min) after removing an electrical load from the battery cell. In Section 2 the experimental procedure is outlined. Results and discussion of the results are presented in Section 3. Finally, the findings of this study are summarized in Section 4.

\section{Experimental methods}

Five commercially available Li-ion cells of three different chemical natures were used as part of this study. These cells were selected based on their power and energy rating. Table 1 summarizes the chemistry, capacity and format of each cell. These cells, representing different groups of Li-ion batteries, i. e., chemistry, power capability and form factors, are currently used in different automotive applications.

In order to isolate the effects of cell relaxation, the temperature and SoC were kept constant for each test; tests were carried out at $25^{\circ} \mathrm{C}$ and $50 \%$ SoC. To precisely adjust the cell SoC to $50 \%$, the cells were discharged at a $1 \mathrm{C}$ rate to the manufacturer's

Table 1: Li-ion cell electrical properties.

\begin{tabular}{rlrll}
\hline Cell number & Chemistry, cathode/anode & Capacity (Ah) & Format & Cell type \\
\hline 1 & LiNiMnCo02(NCM)/LiC6 & 40 & Pouch & Power cell \\
2 & LiFePO4(LFP)/LiC6 & 20 & Pouch & Power cell \\
3 & NCM/LiC6 & 2.2 & Cylindrical & Energy cell \\
4 & NCM/Li4Ti4012(LTO) & 13 & Pouch & Power cell \\
5 & NCM/LiC6 & 3.4 & Cylindrical & Energy cell \\
\hline
\end{tabular}


recommended cut-off voltage, subsequently allowed to rest for 3 hours before being fully recharged according to the manufacturer's recommended charge protocol, using a commercial Li-ion cell cycler. At the end of charging, the cells were allowed to rest for 3 hours prior to being discharged to $50 \%$ SoC at a $1 \mathrm{C}$ rate.

To study the short-term effect of relaxation, measurements were performed in galvanostatic mode using a commercial EIS system. Impedance measurements between $500 \mathrm{mHz}$ and $10 \mathrm{kHz}$ with five frequency points per decade were taken. This frequency range and this number of measurement points were selected as a balance of test duration and number of measurement points. In this setup a measurement will take around 10 seconds. Recording of the EIS data started as soon as the SoC adjustment was completed (i. e., immediately after the $1 \mathrm{C}$ discharge to $50 \%$ SoC), and every minute thereafter.

\section{Results}

The shape of the Nyquist plots at high frequency is similar to that found in the literature. However, at low frequency, cells 1, 2 and 4 show a spiral shape (Fig. 1 (a), (b) and (d)), which has not been reported in previous related literature. The spiral shape in the Nyquist plot starts to shrink from the second test done at $1 \mathrm{~min}$ after SoC adjustment, and finally disappears $10 \mathrm{~min}$ after SoC adjustment when the EIS plot is similar to the Nyquist plots found in the literature [5-8]. The change of shape of the Nyquist plots from $1 \mathrm{~min}$ to $10 \mathrm{~min}$ for cells 1, 2 and 4 happens at a different rate. Cell 3 and 5 (Fig. 1 (c) and (e)) show this spinal shape only for the very first test, which was performed just after the discharge was stopped. Afterward the shape is similar to the Nyquist plots found in the literature. To investigate the experimental setup as a probable cause of the shape, the experiment has been repeated with different conductors, which connect the EIS equipment with the test cell, and even with different sets of EIS equipment; however, in all the instances similar results were found. The spiral shape at the low-frequency end could be related to the measurement time. For a stable EIS measurement, it is essential that there is no change of the cell during measurement at a particular frequency. At the lowest frequency employed $(500 \mathrm{mHz})$, it takes $2 \mathrm{sec}-$ onds to complete the measurement. After discharge was stopped, polarization of the double layer was ceased and as a result cell capacitance and thus total impedance change; this change slows down with the relaxation period. After $10 \mathrm{~min}$ of relaxation the change in the 2-second timescale is probably not significant; however, immediately after discharge it probably is significant, which leads to the spiral shape. With the difference of chemistry, size and form factor, the impedance and frequency response of each cell is different, which could be the root cause of the difference of the spiral shapes. However, for all five cells measured, values of pure Ohmic resistance $\left(R_{o}\right)$ fall 


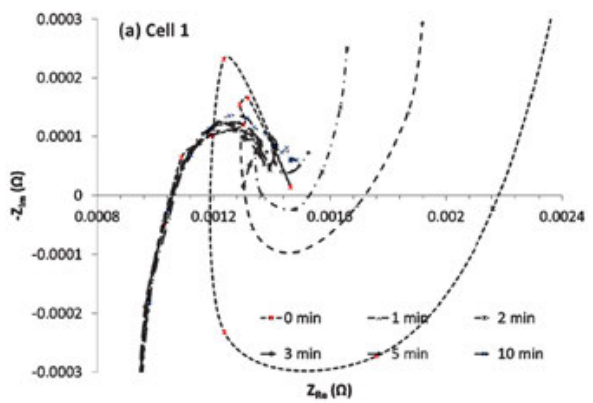

(a)

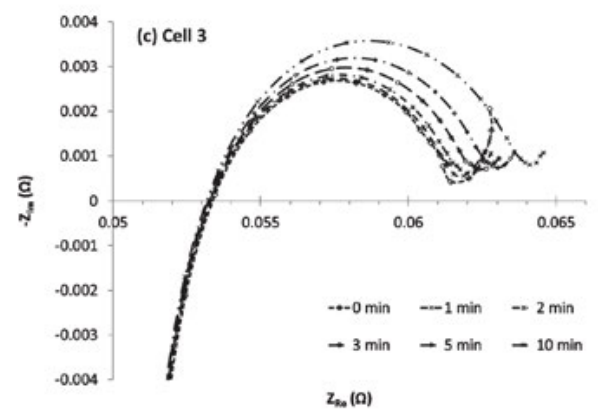

(c)

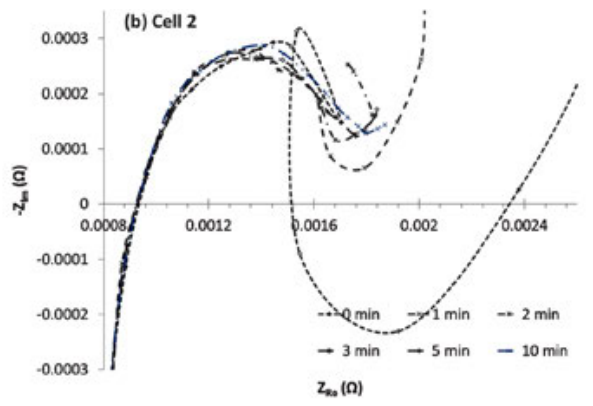

(b)

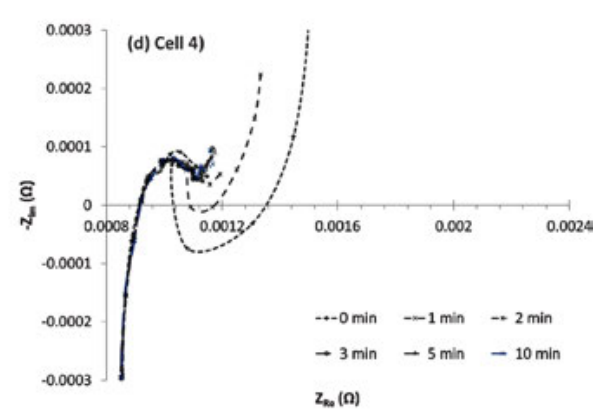

(d)

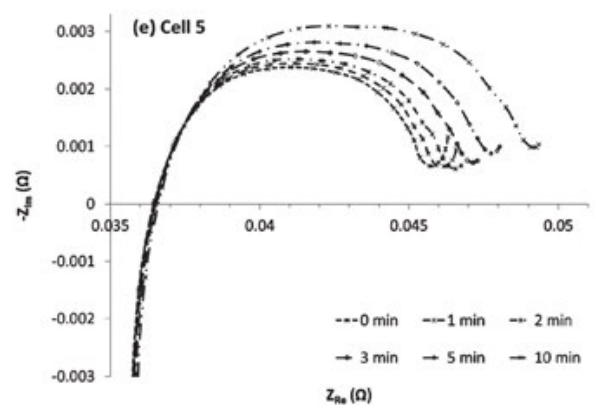

(e)

Fig. 1: Nyquist plots of different cells from 0-10 min.

within experimental error bounds, and therefore they can be considered constant, regardless of relaxation time. This is because $R_{o}$ was found at a frequency higher than $1 \mathrm{kHz}$ for all the cells. Therefore, the measurement time was $1 \mathrm{~ms}$ or shorter and the change of the cell impedance within that short period was not significant enough to affect the measurement. Therefore, $R_{o}$ could be used as online measurement parameter. In contrast to $R_{o}$, the charge transfer resistance and double layer capacitance will be inconsistent within 0-10 minutes after cycling, thus should not be employed for fast measurement. 


\section{Conclusion}

In this research, the variation of cell impedance as a function of relaxation period after removing an electrical load has been investigated for five different commercially available Li-ion cells. It is concluded that the pure Ohmic resistance $R_{o}$ of the cell is independent of the relaxation processes occurring within the cell for the test duration. Therefore, $R_{o}$ could be used for direct comparisons, irrespective of the relaxation period used for the test. The charge transfer resistance and double layer capacitance were found to be inconsistent within 0-10 minutes of relaxation. Therefore, when these two parameters are used as a fast online measurement parameter, they will produce unreliable results, which could affect SoC and SoH estimation. This new knowledge shows the possible inconsistency of SoC and SoH results via an EIS measurement system integrated as part of BMS, which has been proposed by researchers previously.

\section{Bibliography}

[1] E. Barsoukov and J. R. Macdonald, "Impedance Spectroscopy, Theory, Experiment, and Applications", new Jersey: John Wiley \& Sons, 2005.

[2] T. Momma, M. Matsunaga, D. Mukoyama, and T. Osaka, "AC impedance analysis of lithium ion battery under temperature control”, Journal of Power Sources, 2012.

[3] J. P. Schmidt, T. Chrobak, M. Ender, J. Illig, D. Klotz, and E. Ivers-Tiffée, "Studies on LiFePO4 as cathode material using impedance spectroscopy", Journal of Power Sources, 2011.

[4] S. Rodrigues, N. Munichandraiah, and A. K. Shukla, "AC impedance and state-of-charge analysis of a sealed lithium-ion rechargeable battery", Journal of Solid State Electrochemistry, 1999.

[5] A. Eddahech, O. Briat, N. Bertrand, J. Y. Delétage, and J.-M. Vinassa, "Behavior and state-of-health monitoring of Li-ion batteries using impedance spectroscopy and recurrent neural networks", International Journal of Electrical Power \& Energy Systems, 2012.

[6] V. Sauvant-Moynot, J. Bernard, R. Mingant, A. Delaille, F. Mattera, S. Mailley, J.-L. Hognon, and F. Huet, "ALIDISSI, a Research Program to Evaluate Electrochemical Impedance Spectroscopy as a SoC and SoH Diagnosis Tool for Li-ion Batteries”, Oil Gas Sci. Technol-Rev.IFP, 2010.

[7] F. Kindermann, A. Noel, P. Keil, and A. Jossen. "Influence of relaxation time on EIS measurements of Li-ion batteries", In: International Workshop on Impedance Spectroscopy, 2013.

[8] A. Barai, G. H. Chouchelamane, Y. Guo, A. McGordon, and P. Jennings, "A study on the impact of lithium-ion cell relaxation on electrochemical impedance spectroscopy", Journal of Power Sources, 2015. 
